\title{
A Geometrical Analysis of Diffraction Based on Fractal Inhomogeneous Space
}

\author{
Farhad Vedad
}

\section{ABSTRACT}

\begin{abstract}
Although the diffraction of light is a simple experiment in optics, no complete interpretation that satisfactorily describes diffraction in any instance has been developed. Classical physics is unable to describe diffraction phenomena by considering photons solely as particles. In addition, modern mathematical solutions based on the wave-particle duality, including Rayleigh-Sommerfeld diffraction theory, are merely approximations, and fail to provide a model that can be applied to the diffraction caused by both transparent and opaque barriers. This study proposes a diffraction model that can account for both single photons and larger particles, such as electrons, in an inhomogeneous space near the surface of the objects, including the edges of the apertures. Furthermore, a three-dimensional model for calculating the light intensity at any arbitrary observation point is presented. This model provides accurate diffraction simulations and is independent of the near and the far-field zones as well as the aperture material.
\end{abstract}

Keywords: Diffraction, Wave-Particle Duality, Inhomogeneous Space, Fractal Zeta Function, Chirp Function, Rayleigh-Sommerfeld Model.

\section{INTRODUCTION}

The Huygens principle describes wave propagation by treating every point of a wave front as a source of spherical wavelets that produce secondary waves [1]. However, the Huygens principle struggles to provide an adequate explanation for diffraction effects. This was eventually resolved by Miller in 1991, who replaced the spherical point sources with spatiotemporal dipoles [2].

Although, this solution is able to describe phenomena such as refraction, it is unable to explain why the last fringes on either side of the diffraction pattern spread out and lose their fragmental distributions. Furthermore, it fails to explain why the number of fringes on either side of the diffraction pattern a function of the slit width is, i.e., the number of fringes produced with a slit width of $0.01 \mathrm{~mm}$ that can be resolved with the naked eye. However, the number of fringes nearly doubles for a slit width of 0.05 $\mathrm{mm}$. With much larger slits, i.e., a width of $0.35 \mathrm{~mm}$, the number of fringes exceeds 100. Fig. 1(a) and (b) show the diffraction patterns produced using an opaque single slit with a width of $0.05 \mathrm{~mm}$ and $0.35 \mathrm{~mm}$, respectively (wavelength of $532 \mathrm{~nm}$ ).

Moreover, Huygens's solution cannot explain how the width of the diffraction pattern produced by a single slit decrease in response to increasing the thickness of the barriers.
Published Online: January 28, 2021

ISSN: 2684-4451

DOI :10.24018/ejphysics.2021.3.1.36

Farhad Vedad*

Graduate Faculty of Nuclear Engineering and Physics, Amirkabir University of Technology (Tehran Polytechnic), Iran.

(e-mail: farhad.vedad@ gmail.com)

*Corresponding Author

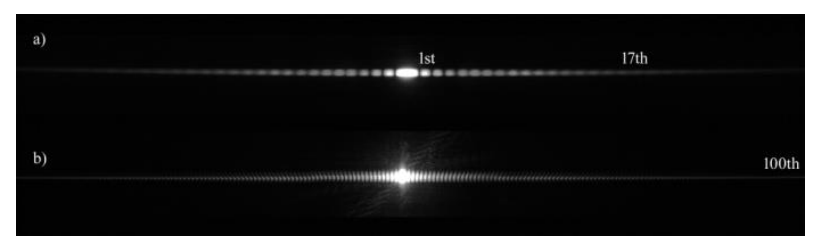

Fig. 1. Diffraction patterns for an opaque single slit with a width of (a) 0.05 and (b) $0.35 \mathrm{~mm}$.

In 1801, Young demonstrated the wave behavior of light via the double-slit experiment, observing a diffraction pattern similar to the interference pattern of the water waves produced by two coherent vibrating sources on the surface of the water [3], [4]. However, this does not seem to be a good example because there are several forces acting between the molecules of the water and between the water and the barriers, including cohesive and adhesive forces, whereas there are no analogous forces between photons.

Later, the concept of wave-particle duality was developed in quantum mechanics as the particle model was unable to provide a unified description for the behavior of both photons and larger particles such as $\mathrm{He}$ atoms at thermal velocity in the double slit experiment. As a result, de Broglie asserted that particles must possess wave-like properties [5]. Reversing the argument, we may also envision photons as particles when considering their detection, reaction, and traveling in free space. Therefore, photons (quanta of energy) can be described as either particles or waves depending on the experiment.

Although the wave-particle concept could easily explain the diffraction caused by an opaque aperture, some arguments were raised by several researchers, including 
Richard Feynman, who called it the only mystery of quantum mechanics [6].

There are several mathematical approximations for diffraction that predict the intensity of light including the Helmholtz-Kirchhoff integral theorem, which considers the solution for a homogenous wave at any point. If $r$ is the length of the vector $\vec{r}$ from the observation point $p$ to any point at the aperture plane, $s$ is the closed surface surrounding $p, \vec{n}$ is the outward normal, $k$ is the wave number equal to $2 \pi / \lambda$ and $U(p)$ is the disturbance at point $p$, the Helmholtz-Kirchhoff theorem can be expressed as [7]:

$$
U(p)=\frac{1}{4 \pi} \iint_{s}\left\{\frac{\partial U}{\partial n}\left[\frac{\exp (i k r)}{r}\right]-U \frac{\partial}{\partial n}\left[\frac{\exp (i k r)}{r}\right]\right\} d s
$$

Alternatively, by simplifying the Huygens-Fresnel principle at the point plying in the $(x, y)$ plane at a distance $z$ normal to the rectangular coordinates lying in the $\left(x_{0}, y_{0}\right)$ plane, we can express the near-field Fresnel approximation:

$$
U(x, y, z)=\frac{e^{i k z}}{i \lambda z} e^{i \frac{k}{2 z}\left(x^{2}+y^{2}\right)} \iint_{-\infty}^{\infty} U\left(x_{0}, y_{0}, 0\right) e^{i \frac{k}{2 z}\left(x_{0}^{2}+y_{0}^{2}\right)} e^{-i \frac{k}{z}\left(x_{0} x+y_{0} y\right)} d x_{0} d y_{0}
$$

Furthermore, for the intensity of light in the far-field, we can use the Fraunhofer diffraction equation [8]:

$$
I(\theta)=I_{0}\left(\sin \left(\frac{k D}{2} \sin (\theta)\right) /\left(\frac{k D}{2} \sin (\theta)\right)\right)^{2}
$$

where $D$ is the distance of the observation point from the aperture and $\theta$ is the angle between the beam and the optical axis. Then, if $\mathrm{w}$ is the width of the rectangular aperture, we can write:

$$
w \sin (\theta)=m \lambda
$$

Finally, owing to certain inconsistencies of the FresnelKirchhoff diffraction integral, we are required to adopt the Rayleigh-Sommerfeld diffraction theory to obtain a more accurate mathematical solution [9]. The RayleighSommerfeld diffraction formulae are used to analyze the propagation of light independently of the Fresnel or Fraunhofer approximations; instead, they use the angular spectrum and direct integration methods [10].

Aside from wave-particle duality, none of these mathematical solutions can explain why the central band of the far-field diffraction pattern is a minimum for diffraction by a transparent single slit, or why it becomes significantly narrower if the barriers are semitransparent as opposed to an opaque single slit [11].

Such examples require the pursuit of a better solution that can explain diffraction phenomena independent of the aperture material and, preferably, independent of the nearand far-field zones.

\section{ANALYTICAL METHOD}

Let us assume that the space inside the aperture is homogenous with a constant refractive index. In this case, the concept of wave-particle duality represents the best solution for calculating the intensity of light at any arbitrary observation point, which leads us to consider equations (1) and (2), or the Rayleigh-Sommerfeld diffraction formulae, although they are valid solely for the opaque barriers.

Alternatively, as summing that the space is inhomogeneous close to the surfaces of the obstacles, as well as the edges of the aperture, the refractive index near these surfaces will be higher, and will reduce as the distance from the object is increased.

Considering this assumption alongside Snell's law and the plane wave aberrations for a diffraction cone produced by an opaque wedge (see Rubinowicz for details [12]), whereby the edge-diffracted rays satisfy Fermat's principle, leads us to another perspective. The goal of this new concept is to produce a diffraction model that works independently of the aperture material and its transparency or reflectivity in both the near and far-fields.

To achieve this, I performed diffraction experiments with opaque, transparent, and semitransparent apertures and compared the results with three-dimensional simulations. First, I photographed the diffraction pattern millimeter by millimeter along the optical axis from the plane of the aperture to the Fraunhofer zone and combined the images.

All experiments were performed using an opaque single slit with a width of $0.35 \mathrm{~mm}$ because larger apertures extend the near-field zone and produce more fringes. Then, I stitched the images together to reconstruct the entire diffraction image and analyzed the ray paths using Autodesk Alias to produce a three-dimensional digital model of the diffraction pattern.

\section{TREATMENT OF DIFFRACTION PATHS}

Fig. 2 shows the diffraction pattern produced by a single slit width of $0.35 \mathrm{~mm}$ from $Z=3 \mathrm{~mm}$ to $Z=56 \mathrm{~mm}$, where $Z$ is the distance from the plane of the aperture along the direction normal to the aperture.

As shown in Fig. 2, the definition of the near and farfields are not correct because point $\mathrm{A}$, which is closer to the aperture compared to point $\mathrm{B}$, exhibits a ray similar to the far-field, while point B exhibits near-field characteristics.

Furthermore, calculating the light intensity within the triangle formed by the points $\mathrm{M}, \mathrm{Q}$, and $\mathrm{P}$ because the rays out of that triangle on either side are straight.

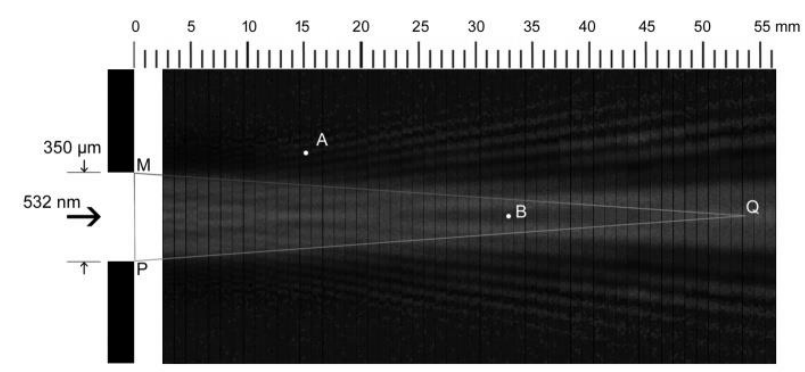

Fig. 2. Image of the diffraction pattern in the near and far-fields produced by an opaque aperture with a width of $0.35 \mathrm{~mm}$. 
Although Fig. 2 appears similar to the intensity distribution of the Fresnel diffraction pattern [13], they are not the same. This difference can be seen by magnifying an image of a Fresnel diffraction pattern by a factor of 10 along the $z$-axis, as shown in Fig. 3(a). Here, the light rays emerging from the triangle MQP are curved, as shown by the black arrows. However, the paths in Fig. 2 are straight.

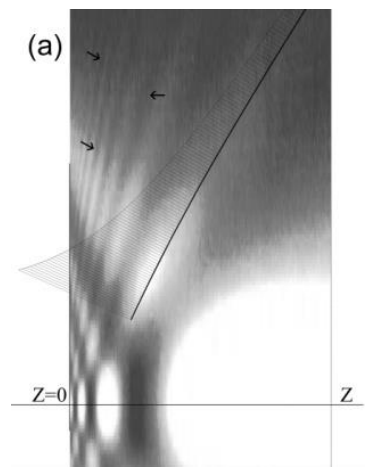

(b)

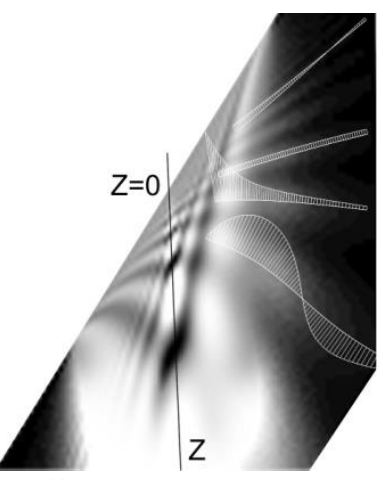

Fig. 3 (a). Magnified image of Fresnel diffraction pattern showing ray path curvature (black arrows). (b) Near-field diffraction pattern modeled using the Rayleigh-Sommerfeld model.

Next, I simulated the propagation of the near-field diffraction pattern based on the Rayleigh-Sommerfeld formulae using MATLAB [14]. Then, I magnified the resulting image non-proportionally by a factor of 10 along the $z$-axis. I rotated the image until one of the paths outside triangle MQP remained almost horizontal. Then, I magnified it again non-proportionally by a factor of 10 along this path. Fig. 3(b) shows the curvature analysis of the paths produced in MATLAB. As this figure shows, the paths are not straight and have different curvatures.

Fig. 4 indicates that the lines lie on the diffraction paths. As this figure shows, the paths are straight from $Z_{0}$ to any point in the far-field, and the distribution of paths at $\mathrm{Z}_{0}$ resembles up-chirped functions. However, one path, $\overline{A_{0} B_{0}}$, does not follow this trend. To study this path, further experiments and analysis are required. Therefore, the other paths are considered first.

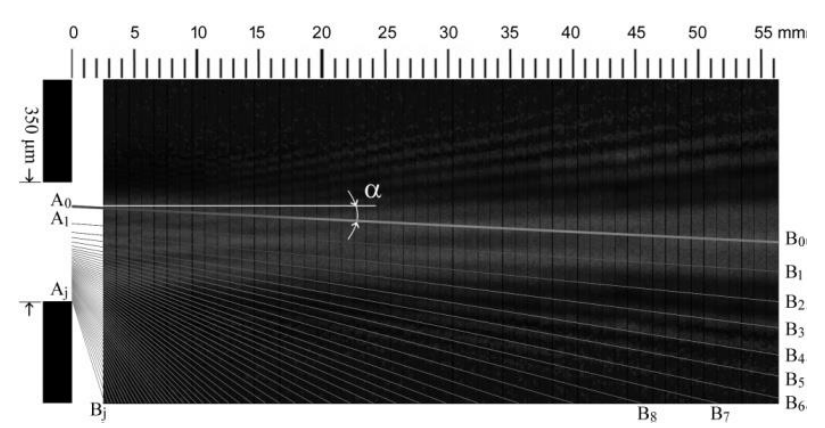

Fig. 4. Distribution of the diffraction paths.

It should be noted that angle $\alpha$ in Fig. 4 was measured as approximately $8.76 \times 10^{-4 \circ}(\sim 3.15$ arcseconds $)$ for a wavelength of $532 \mathrm{~nm}$ and a single opaque slit with a width of $0.35 \mathrm{~mm}$. This changed to $5.33 \times 10^{-3 \circ} \quad(\sim 19.188$ arcseconds) for a single slit with a width of $0.050 \mathrm{~mm}$. As each path behaves independently of the others, the intensity of light along each path from $\mathrm{A}_{\mathrm{m}}$, at the aperture plane, to $\mathrm{B}_{\mathrm{m}}$, at any distance from the aperture, stays equal. Geometrically, the area of every ray cross section from $Z_{0}$ to any far-field point is equal, as shown in Fig. 5.

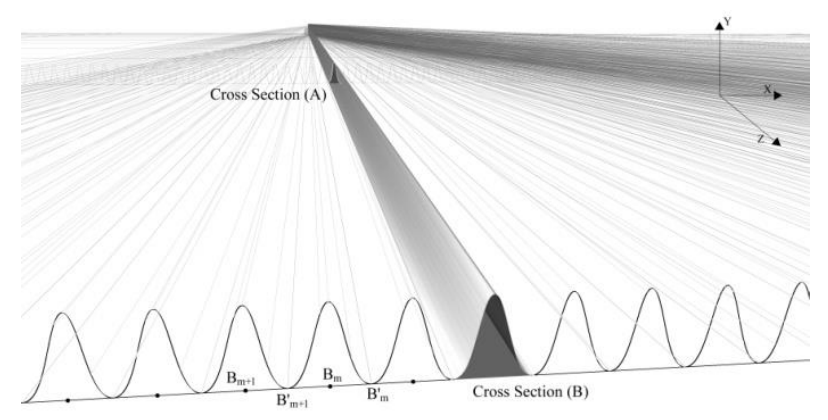

Fig. 5. Diagram illustrating ray cross sections in the far-field.

Conversely, from $\overline{A_{0} B_{0}}$ to $\overline{A_{j} B_{j}}$, the ray intensity decreases. It should be noted that the points $\mathrm{A}_{0}$ to $\mathrm{A}_{\mathrm{j}}$ at $\mathrm{Z}_{0}$ were arranged on a plane to simplify the calculation. As mentioned before, at $\mathrm{Z}_{0}$, the frequency of the distribution points $A_{0}$ to $A_{j}$ varies along the $x$-axis with a geometric relationship over the distance from the edge of the aperture. Thus, they can be considered as up-chirped functions in two ways. First, by as summing that if $\Phi(x)$, the instantaneous frequency is a linear function with an initial frequency of $\Phi_{0}$, which results in the following time-dependent linear chirped function [15]:

$$
\phi(t)=\phi_{0}+k t, \text { and } x(t)=A \sin \left(2 \pi \int_{0}^{\tau} \Phi(t) d t\right)
$$

Then the Fourier transform is given by:

$$
F[x(t)]=\int_{0}^{T} \sin \left(\omega_{0} t+\pi k t^{2}\right) \exp (-i \omega t) d t
$$

This is a general form of the equation with a geometric relationship over time. Later, this equation needs to be adjusted to follow a geometric relationship over the distance. Furthermore, the geometric $(\alpha, \beta)$-chirp of the planar set $\mathrm{A}$ is defined by $\mathrm{A}_{\mathrm{k}}$, which is the Cartesian product of the one-point set $\left\{k^{-1 / \beta}\right\}$ and the open interval $\left(0, k^{\alpha / \beta}\right)$ [16]:

$$
A=\bigcup_{k \geq 1} A_{k}, \quad A_{k}=\left\{k^{-1 / \beta}\right\} \times\left(0, k^{-\alpha / \beta}\right)
$$

This enables the fractal zeta functions of the inhomogeneous refracted space to be described, with $n>1$ near the surface of the barrier and $n=1$ when $x$ approaches infinity if the medium is a vacuum. Here, $s$ is a complex number with a sufficiently large real part, and $\zeta_{A}(s)$ is the distance zeta function of $\mathrm{A}$, where $\mathrm{A}$ is an arbitrary compact subset of the Euclidian space $\mathbb{R}^{N} . k$ is the languidity exponent, and $\mathrm{R}(\mathrm{s})$ is the reminder term equal to the zeta function of A corresponding to the boundary of a distance set.

$$
\zeta_{A}(s)=\sum_{k=1}^{\infty} \zeta_{k}(s)+R(s)
$$


Furthermore, each fractal of the refracted space behaves as a refractive element in which the light can be considered via ray tracing. Because the refractive index of the last elements is nearly the same as the refractive index of the medium ( $n=1$ for a vacuum), the outermost fringes of the diffraction pattern are washed out by each other and become stretched (see Fig. 6). As mentioned before, the Huygens principle is unable to describe this behavior.

This set can be a complicated geometric imitation of the variables-chirped wave in three-dimensional Euclidean space, $\mathbb{R} 3$. Fig. 4 displays the simplified form in $\mathbb{R} 2$.

The second assumption is that the fractal elements follow a nonlinear geometric $(\alpha, \beta)$-chirp.

The spatial variance of chirp is related to the map function and, if the map function uses log-polar coordinates, the chirp behaves exponentially [17]. This is equivalent to the distribution of points $\mathrm{A}_{0}$ to $\mathrm{A}_{\mathrm{j}}$ along the $x$-axis on the aperture plane in Fig. 4. As this is similar to Fig. 6, it represents an exponential up-chirp due to the changing rate of $\overline{A_{m} A_{m-1}} / \overline{A_{m+1} A_{m}}$.

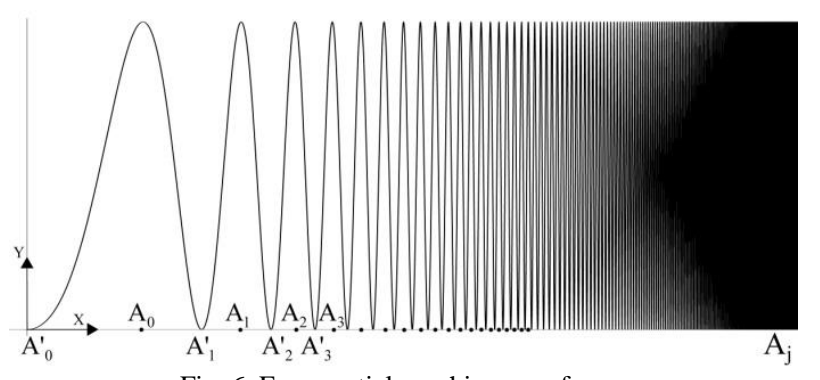

Fig. 6. Exponential up-chirp waveform.

As shown in Fig. 6, the frequency of the sinusoidal signal of the up-chirp waveform increases with distance. Therefore, the signal frequency should be an exponential function of the distance, which can be expressed as:

$$
g(x)=\sin ^{2}\left(e^{f(x)}-1\right)
$$

where $f(x)$ is the geometrical function related to the dimensions and the shape of the aperture, and the coordinate is located at the aperture surface.

Furthermore, if $g(x)$ is defined by Eq. (9), $\overline{A_{0}^{\prime}}$ to $\overline{A_{m}^{\prime}}$ are minima at $\mathrm{Z}_{0}, \overline{B_{0}^{\prime}}$ to $\overline{B_{m}^{\prime}}$ are minima at $\mathrm{Z}$, and $I(x)$ is the distribution function of the light intensity in the far-field along the $x$-axis, then the total light intensity between two minima, at any arbitrary cross section, will be equal, as shown in Fig. 5. This is expressed by:

$$
\int_{A_{m}^{\prime}}^{A_{m+1}^{\prime}} g(x) d x=\int_{B_{m}^{\prime}}^{B_{m+1}^{\prime}} I(x) d x
$$

which predicts an extremely high intensity amplitude at $\mathrm{Z}_{0}$ compared to any arbitrary observation point because of the extremely narrow bandwidth, as described by Eq. (11):

$$
\left\{\begin{array}{l}
I_{\max }\left(A_{m}\right) \gg I_{\max }\left(B_{m}\right) \\
\overline{A_{m}^{\prime} A_{m+1}^{\prime}} \gg \overline{B_{m}^{\prime} B_{m+1}^{\prime}}
\end{array}\right.
$$

The experimental results indicate that the diffraction pattern can be revived even with a single arrival photon, if the exposure time is adequate. This concept can easily demonstrate how the arrival photons will follow the paths, which are depicted in Fig. 4, based on their arrival point with their specific refractive indices relevant to the fractal inhomogeneous space in the plane of the aperture and their own wavelengths.

In addition, it should be noted that if we divide both sides of Eq. (4) by $\lambda$, then the left-hand side is analogous to Snell's law, where $\lambda_{0}$ and $\theta_{0}$ refer to the last incident ray inside the aperture that exits into the inhomogeneous refracted space:

$$
\frac{\sin (\theta)}{\lambda}=\frac{m}{w}, \quad \frac{\sin (\theta)}{\lambda}=\frac{\sin \left(\theta_{0}\right)}{\lambda_{0}}=\frac{m}{w}
$$

\section{PROPAGATION OF DIFFRACTED RAYS}

As mentioned before, diffraction experiments with opaque, transparent, and semitransparent aperture materials need to be performed to determine the structure of the path $\overline{A_{0} B_{0}}$. Therefore, the rays $\overline{A_{1} B_{1}}$ to $\overline{A_{j} B_{j}}$ are considered initially. To simplify the analysis, only sixty rays (which can be termed refracted rays) corresponding to sixty fringes in the far-field (i.e., up to $\overline{A_{60} B_{60}}$ ) were considered. In addition, the following steps were implemented:

1. The path $\overline{A_{0} B_{0}}$ on either side of the symmetrical diffraction model was initially ignored.

2. The positions of points $A_{0}$ to $A_{j}$ at the aperture plane and the points $B_{0}$ to $B_{j}$ at the far-field plane were identified.

3. Profiles of the light intensity relevant to $\overline{A_{m} B_{m}}$ based on Eq. (10) were drawn.

4. These profiles were duplicated to provide a symmetrical model (see Fig. 7).

5. To consider the paths in their entirety from $Z_{0}$ to the observation screen in the far-field, it is necessary to scale down the model non-proportionally along the optical axis ( $z$ axis) because the width of the aperture is normally about a fragment of millimeter (i.e., a single slit with a width of 0.35 $\mathrm{mm}$ in Fig. 2), whereas the length of paths $\overline{A_{m} B_{m}}$ relevant to the far-field are very long (here, it is $1 \mathrm{~m}$ ).

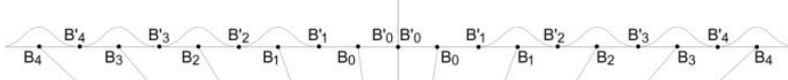

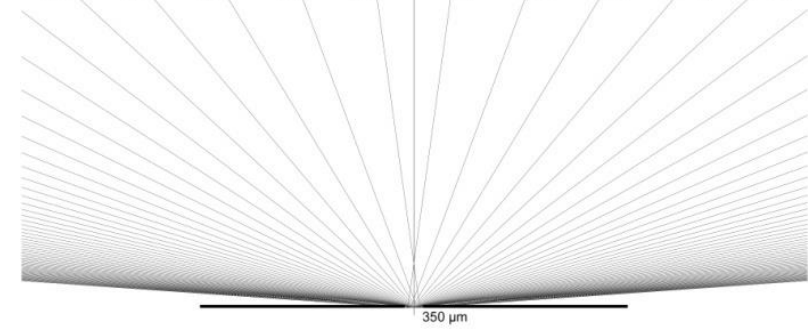

Fig. 7. Distribution of the refracted rays on either side of the diffraction pattern, excluding the rays that may produce the central band.

Although the paraxial approximation, which is a smallangle approximation to the optical axis, is used in Gaussian optics and ray tracing in geometric optics [18], [19], it is not necessary to apply it here because the relevant minima and 
maxima at $\mathrm{A}_{\mathrm{m}}$ and $\mathrm{B}_{\mathrm{m}}$ are connected.

It is important to note that the length of $\overline{A_{59} A_{60}}$ was measured to be approximately $1100 \mathrm{~nm}$ for $\mathrm{w}=0.35 \mathrm{~mm}$ (see also Fig. 6).

\section{Definition of the Path $\overline{A_{0} B_{0}}$}

To complete Fig. 7 , the diffraction pattern created by opaque and transparent barriers must be analyzed to determine the differences and similarities.

The diffraction experiments with transparent and semitransparent apertures show that the main functional parameter is the refractive index contrast between the aperture and the medium. Fig. 8 shows the single-slit diffraction pattern created by a glass single slit with a width of $0.1 \mathrm{~mm}$ and thickness of $0.170 \mathrm{~mm}(n=1.515)$ at a distance of $8 \mathrm{~m}$, which illustrates the absence of a constructive interference fringe coinciding with the optical axis.

It should be noted that a narrow bright central band may appear in the far-field by changing the refractive index contrasts between the aperture and the medium, for example, in the experiment of a glass $(n=1.515$, thickness $=0.170 \mathrm{~mm}$ ) single slit with a width of $0.1 \mathrm{~mm}$ at $20^{\circ} \mathrm{C}$ inside aniseed essential oil $(n=1.5385)$ for a 635 -nm laser, and also in the experiment with a glass $(n=1.515$, thickness $=0.192 \mathrm{~mm}$ ) single slit with a width of $0.1 \mathrm{~mm}$ at $27{ }^{\circ} \mathrm{C}$ inside rapeseed oil $(n=1.4705)$ for a 532-nm laser [11].

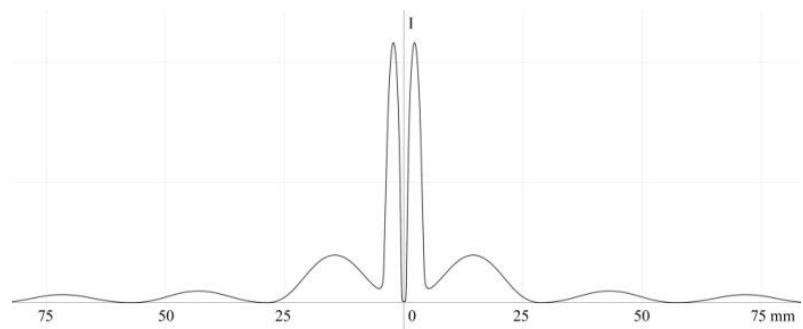

Fig. 8. Illustration of the far-field single-slit diffraction pattern for a glass aperture.

Fig. 9 shows the single-slit diffraction pattern created by a fused quartz aperture at a distance of $8 \mathrm{~m}$, for which the transparency at the edges of the fused quartz slit is significantly less than that through the center of the barrier, where it exceeds $90 \%$. In this experiment, a fused quartz single slit with a width of $100 \mu \mathrm{m}$ and thickness of $0.17 \mathrm{~mm}$ is employed $(n=1.4658(447.1 \mathrm{~nm}), 1.4607(532 \mathrm{~nm})$, $1.4570(632.8 \mathrm{~nm})$, clear aperture $(\%): \geq 90)[11]$.

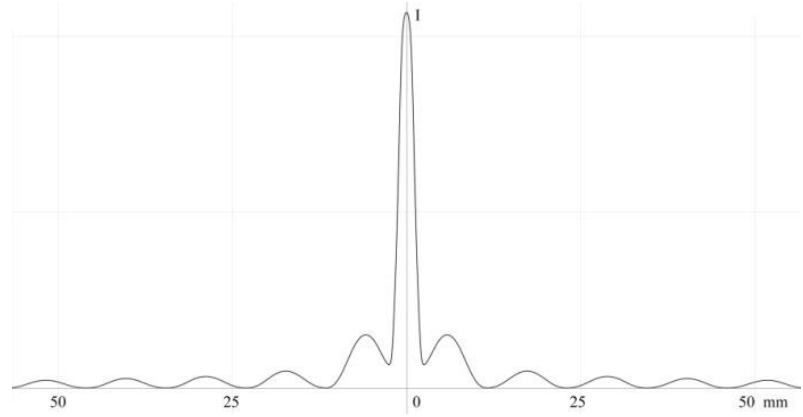

Fig. 9. Illustration of the far-field single-slit diffraction pattern for a fused quartz aperture.
Furthermore, Fig. 10 shows the single-slit diffraction pattern created by an opaque aperture (302 stainless steel with black oxide finish) at a distance of $8 \mathrm{~m}$ (for a slit width of $100 \mu \mathrm{m}$ and thickness of $0.127 \mathrm{~mm}$ ).

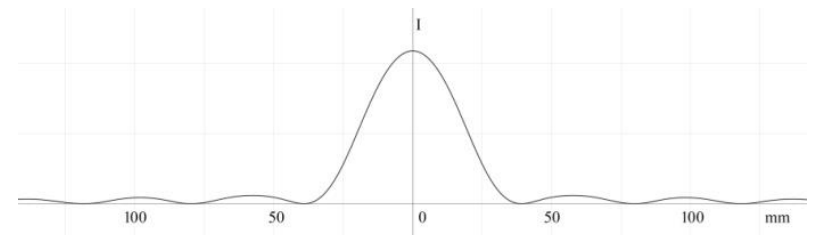

Fig. 10. Illustration of the far-field single-slit diffraction pattern for an opaque aperture.

Comparing Fig. 8, 9, and 10 demonstrates that the principal visual difference in the diffraction patterns occurs at the central ray corresponding to $\overline{A_{0} B_{0}}$, while the other fringes on either side of the diffraction pattern are similar.

The experiments involving transparent and semitransparent barriers suggest that the central band of the diffraction pattern is produced by two kinds of rays: a refracted ray similar to those in Fig. 7 and its duplicate owing to the symmetry of the model. Subsequently, we may consider Fig. 8 from another perspective, as shown in Fig. 11 , in which it is clear that the numerical sum of the intensities of the refracted and reflected rays is shown in Fig. 8.

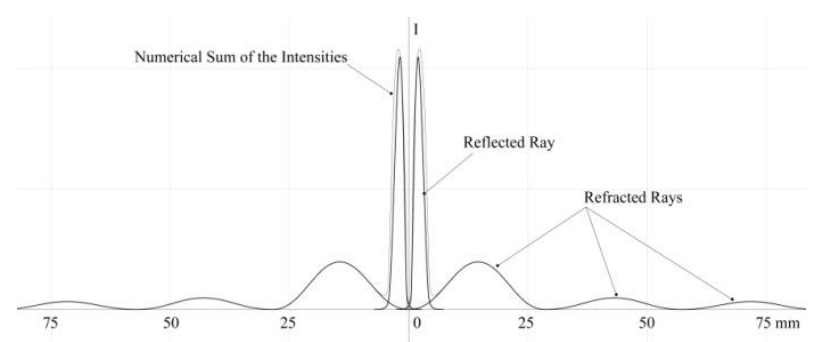

Fig. 11. Central band of the far-field diffraction pattern for a glass aperture.

This assumption arose when I turned a convex mirror (diameter: 25mm, focal length: $-7.75 \mathrm{~mm}$ ) around the straight edge of a thin glass sheet $(n=1.515$, thickness $=0.192 \mathrm{~mm}$ ) while considering the diffraction pattern.

Similarly, Fig. 9 shows that, as illustrated in Fig. 12, the numerical sum of the intensities of the reflected and refracted rays and their symmetrical counterparts in the center of the diffraction pattern produce the central single narrow band.

Therefore, this model can be used to evaluate the effects of aperture transparency and reflectivity.

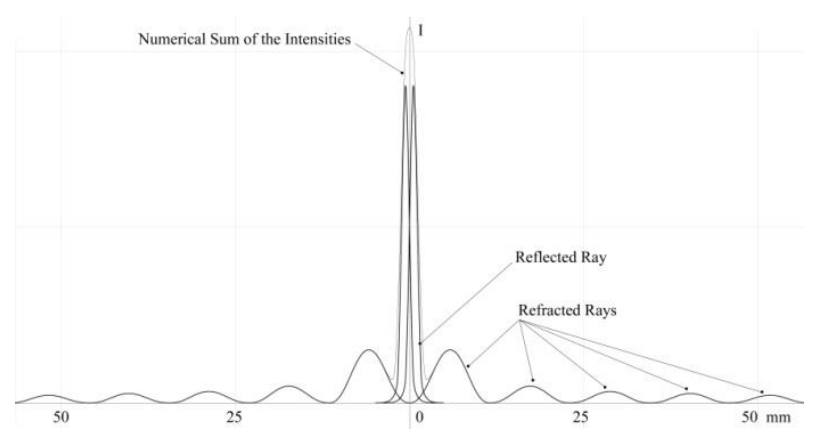

Fig. 12. Central band of the far-field diffraction pattern for a fused quartz aperture. 
Finally, we can follow the same logic to examine the central band of the diffraction pattern produced by an opaque aperture, as shown in Fig. 13. In this case the maximum light intensity at the center of the diffraction pattern is the combination of the refracted and reflected rays with their symmetrical duplicates and is equal to their combined numerical sum.

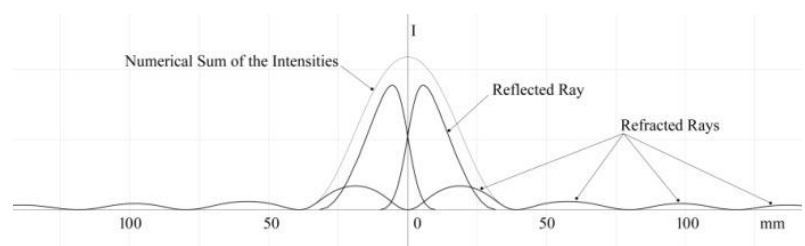

Fig. 13. Central band of the far-field diffraction pattern for an opaque aperture.

\section{THREE-DimenSIONAL DIFFRACTION MODEL}

To produce a three-dimensional diffraction model to predict the intensity of light in any arbitrary point in the $x z$ plane, two generation curves and two rails for each profile of the refracted rays are required (see Fig. 5 and 7). As shown by Fig. 13, producing the profile of the reflected ray and the central refracted ray for the opaque barrier is easy because they overlay each other. It is important to note that for the transparent and semitransparent barriers, these two profiles should be generated separately. This is because the profiles are duplicated to make the model symmetrical only if it is relevant and, if not, i.e., when the laser beam is not perfectly perpendicular to the plane of the aperture, then each side of the model is considered separately.

The rails of each profile can be defined by the connecting lines of the intensity minima, which correspond to $\overline{A_{m}^{\prime} B_{m}^{\prime}}$ in Fig. 6 and 7.

The generation curves should respect the light intensity in the domain of $\overline{A_{m}^{\prime} A_{m+1}^{\prime}}$ at the aperture plane and $\overline{B_{m}^{\prime} B_{m+1}^{\prime}}$ at the far-field observation plane, as described in Eq. (10), and the equivalence of the ray cross sections.

The far-field generation curves can be determined experimentally using a detector to scan the diffraction pattern at various distances from the aperture. Then, Eq. (10) can be used to define the corresponding intensity maximum from $Z_{0}$ to $Z$ at any arbitrary distance from the aperture.

Fig. 14 shows the top view of the three-dimensional model based on the definition of one reflected ray and sixty refracted rays (plus an additional central refracted ray). In this model, the width of the aperture was set to $0.35 \mathrm{~mm}$ and the distance of the observation screen to the aperture was set to $1 \mathrm{~m}$. Therefore, it is necessary to scale down the model non-proportionally along the optical axis.

As Fig. 14 shows, the three-dimensional model, in the top view, matches perfectly with the experimental result shown in Fig. 2.

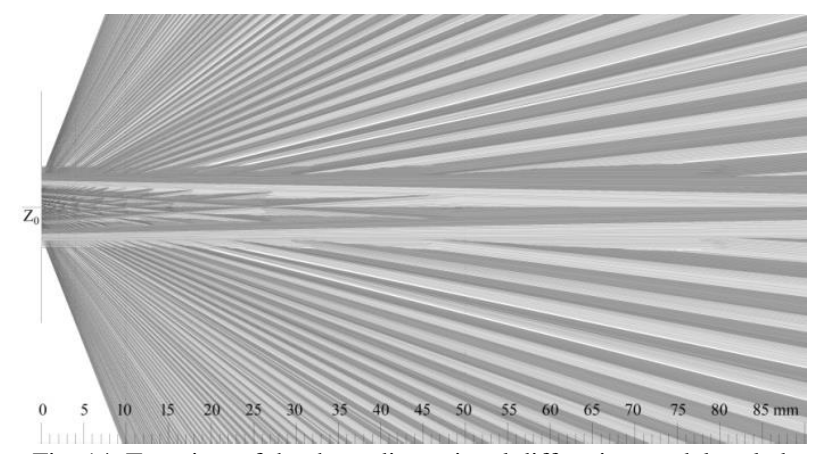

Fig. 14. Top view of the three-dimensional diffraction model scaled non-proportionally along the optical axis.

Now, sections can be made at any arbitrary distance from the aperture to measure the intensity of light at any arbitrary point by calculating the sum of the amplitudes. From Fig. 15 , it can be seen that two generation curves exist at $Z=1 \mathrm{~m}$, each of which is the numerical sum of the reflected ray and the central refracted ray (for the opaque aperture). Furthermore, the amplitude of the intensity at $\mathrm{A}_{0}$ is much greater than the intensity at $\mathrm{B}_{0}$, in accordance with Eq. (11).

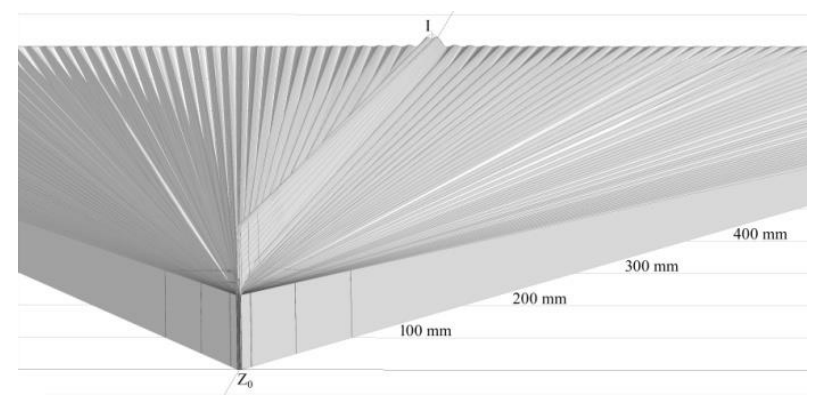

Fig. 15. Orthographic view of the three-dimensional diffraction model.

As Fig. 16 shows, the central band of the diffraction pattern is produced by four rays, a reflected and a refracted ray, and their duplicates.

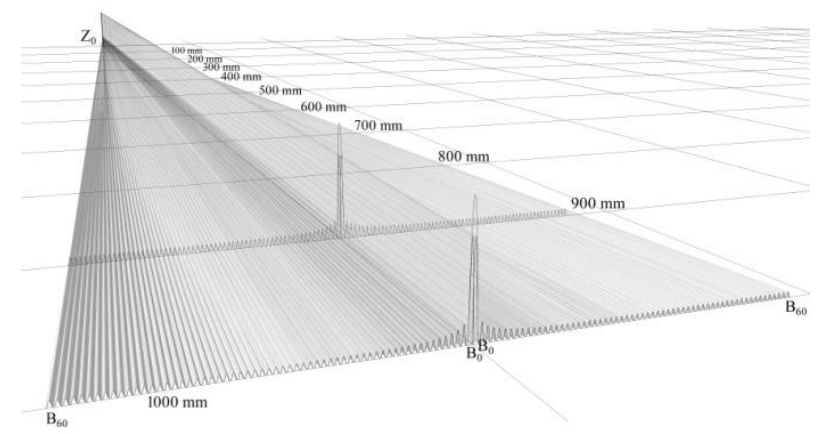

Fig. 16. The perspective view of the 3D digital model of diffraction

Finally, the model is ready for sectioning. Fig. 17 shows four sections at $1-\mathrm{mm}$ intervals from the aperture alongside the light intensity distribution at the aperture for each interval.

The sections in the three-dimensional model appear similar to those at distances of 2 and $4 \mathrm{~mm}$, and their numerical sum is similar to those sections at distances of 1 and $4 \mathrm{~mm}$ from the aperture in Fig. 17. 


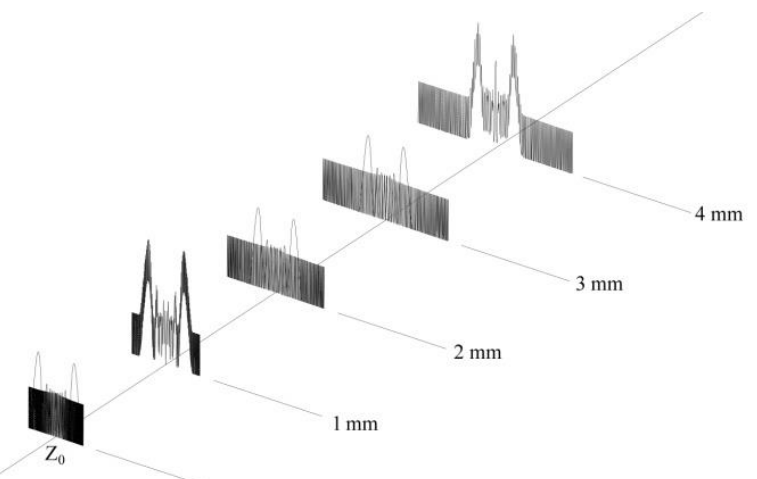

Fig. 17. Sections of the three-dimensional diffraction model.

It is clear that, for the different incident wavelengths for a similar aperture, the only model variable liable to change is the angle of the rays relevant to Snell's law. Moreover, this concept provides the opportunity to model the diffraction patterns produced by apertures of any kind of material or shape in the same structure. The concept starts by indicating points $\overline{A_{0}^{\prime}}$ to $\overline{A_{m}^{\prime}}$ on the plane of the aperture and points $\overline{B_{0}^{\prime}}$ to $\overline{B_{m}^{\prime}}$ on the plane in the far-field, and then, producing the profiles of the refracted and reflected rays.

\section{CONCLUSION}

Through considering the cross sections of the threedimensional diffraction model it is demonstrated that the paths of photons and other larger particles adhere to Fermat's principle and, furthermore, it can be deduced that the space near the surface of an object refracts light inhomogeneously, in accordance with a variable refractive index described by an exponential up-chirp function. Therefore, the wave-particle duality of light and other larger particles is not responsible for diffraction.

The diffraction pattern is a combination of refracted and reflected rays, with the central band resulting from the combination of reflected and refracted rays in a symmetrical system.

Furthermore, the number of fringes on either side of the diffraction pattern is small for smaller apertures and large for larger apertures. In addition, the outermost regions of the diffraction patterns stretch because the last fractal elements of the inhomogeneous space in the aperture plane maintain a refractive index that is very close to the original refractive index.

The properties of the aperture edges, including their transparency and reflectivity, as well as the thickness of the aperture, influence the appearance of the diffraction pattern.

Finally, the accurate three-dimensional diffraction model based on this concept of space being refracted near the surface of an object and its inhomogeneous refractive index enables the diffractive behavior of all shapes of transparent and opaque apertures to be modeled independent of the near and far-field zones for symmetrical and asymmetrical patterns.

\section{REFERENCES}

[1] Olaf Stenzel, Optical Coatings: Material Aspects in Theory and Practice, Springer-Verlag Berlin Heidelberg, 2014, pp. 335-340.

[2] David A. B. Miller, Huygens's wave propagation principle corrected, Optics letters, Vol. 16, No. 18, 1991, pp.1370.

[3] Raymond A. Serway and John W. Jewett, Physics for Scientists and Engineers, Volume 2, Edition 7, Thomson Learning, 2008, pp.1053.

[4] Michele Bonazzi and Valentina Di Simone, Redesigning Worldwide Connections, Cambridge Scholars Publishing, 2015, pp. 157.

[5] Alwyn van der Merwe, and Augusto Garuccio, Waves and Particles in Light and Matter, Springer Science+Business Media, 1994, pp. 34-35.

[6] Feynman, Richard P.; Robert B. Leighton; Matthew Sands. The Feynman Lectures on Physics, Vol. 3. Basic Books, 2011, New York. Chap. 2, 3, and 4.

[7] Joseph W. Goodman, Introduction to Fourier Optics, W. H. Freeman and Company, 2017, Chap. 2 and 3.

[8] Subhasish Dutta Gupta, Nirmalya Ghosh, and Ayan Banerjee, Wave Optics: Basic Concepts and Contemporary Trends, CRC Press, 2016, pp.143-147.

[9] Bernard C. Kress and Patrick Meyrueis, Applied Digital Optics: From Micro-optics to Nanophotonics, John Wiley \& Sons, 2009, pp. 592.

[10] Fabin Shen and Anbo Wang, "Fast-Fourier-transform based numerical integration method for the Rayleigh-Sommerfeld diffraction formula," Appl. Opt. 45, 1102-1110 (2006).

[11] Vedad, F. (2020). Experimental Investigation of Diffraction caused by Transparent Barriers. European Journal of Applied Physics, 2(5). ISSN: 2684-445, September 19, 2020.

[12] Pyotr Ya. Ufimtsev, Fundamentals of the Physical Theory of Diffraction, Second Edition, John Wiley \& Sons, Inc., 2014, pp.114118.

[13] Min Gu, Advanced Optical Imaging Theory, Springer Series in Optical Sciences, Vol. 75, Springer Science \& Business Media, 2000, pp. 30.

[14] Gillen, Glen \& Guha, Shekhar. (2004). Modeling and Propagation of Near-Field Diffraction Patterns: A More Complete Approach. Physics. 72. 10.1119/1.1767102.

[15] Ian Sharp and Kegen Yu, Wireless Positioning: Principles and Practice, Springer Nature Singapore Pte Ltd, 2019, pp. 45-48.

[16] Michel L. Lapidus, Goran Radunović, and Darko Žubrinić, Fractal Zeta Functions and Fractal Drums: Higher-Dimensional Theory of Complex Dimensions, Springer, 2017, pp. 229-231.

[17] Giorgio Bonmassar and Eric L. Schwartz, Space-Variant Fourier Analysis: The Exponential Chirp Transform, Boston University Center for Adaptive Systems and Department of Cognitive and Neural Systems, 1995.

[18] Frank Träger, Springer Handbook of Lasers and Optics, Springer New York, 2007, pp. 41-60.

[19] K. K. Sharma, OPTICS: Principles and Applications, Elsevier Inc., 2006, pp. 159-161.

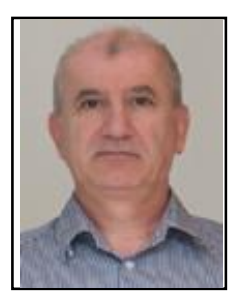

Farhad Vedad was born in Mashhad, Iran in 1963. After finishing his BSc in atomic applied physics at Tehran polytechnic university in 1994, he continued environmental engineering for a master's degree. Mr. Farhad Vedad set up a private physics lab for modern optics and meanwhile he is the author of more than twenty original articles and four books including "The Maps of Balkhi" which is trying the physics methods to solve a historical geography subject which is published by Society for the National Heritage of Iran and Experimental Investigation of Diffraction caused by Transparent Barriers. European Journal of Applied Physics, 2(5). ISSN: 2684-445, September 19, 2020. 